\title{
A hypomorphic mutation in Pold1 disrupts the coordination of embryo size expansion and morphogenesis during gastrulation
}

\author{
Tingxu Chen ${ }^{1,3}$, Heather Alcorn ${ }^{1}$, Sujan Devbhandari², Dirk Remus ${ }^{2}$, Elizabeth \\ 5 Lacy ${ }^{1}$, Danwei Huangfu ${ }^{1,}{ }^{*}$, Kathryn V. Anderson ${ }^{1, \dagger}$ \\ ${ }^{1}$ Developmental Biology Program, Sloan Kettering Institute, Memorial Sloan Kettering Cancer \\ Center, New York, NY 10065, USA. \\ ${ }^{2}$ Molecular Biology Program, Sloan Kettering Institute, Memorial Sloan Kettering Cancer \\ 10 Center, New York, NY 10065, USA. \\ ${ }^{3}$ Louis V. Gerstner Jr. Graduate School of Biomedical Sciences, Memorial Sloan Kettering \\ Cancer Center, New York, NY 10065, USA. \\ † Deceased. \\ * Correspondence to: Danwei Huangfu (huangfud@mskcc.org)
}

Keywords: Pold1, cell proliferation, gastrulation, embryo size, morphogenesis, lineage specification 
bioRxiv preprint doi: https://doi.org/10.1101/2022.03.07.483315; this version posted March 7, 2022. The copyright holder for this preprint (which was not certified by peer review) is the author/funder. All rights reserved. No reuse allowed without permission.

\section{Summary statement}

Pold1 hypomorphic mutation caused reduced size and abnormal morphology of gastrulating mouse embryos, supporting the importance of coordinated embryo size, lineage specification and tissue morphogenesis for normal embryogenesis. 


\section{Abstract}

Formation of a properly sized and patterned embryo during gastrulation requires a wellcoordinated interplay between cell proliferation, lineage specification and tissue morphogenesis. Following transient physical or pharmacological manipulations, pre-gastrulation stage mouse embryos show remarkable plasticity to recover and resume normal development. However, it remains unclear how mechanisms driving lineage specification and morphogenesis respond to defects in cell proliferation during and after gastrulation. Null mutations in DNA replication or cell-cycle related genes frequently lead to cell cycle arrest and reduced cell proliferation, resulting in developmental arrest before the onset of gastrulation; such early lethality precludes studies aiming to determine the impact of cell proliferation on lineage specification and morphogenesis during gastrulation. From an unbiased ENU mutagenesis screen, we discovered a mouse mutant, tiny siren (tyrn), that carries a hypomorphic mutation producing an aspartate to tyrosine (D939Y) substitution in Pold1, the catalytic subunit of DNA polymerase $\delta$. Impaired cell proliferation in the tyrn mutant leaves anterior-posterior patterning unperturbed during gastrulation but results in an overall reduction in embryo size and in severe morphogenetic defects. Our analyses show that the successful execution of morphogenetic events during gastrulation requires that lineage specification and the ordered production of differentiated cell types occur in concordance with embryonic growth. 


\section{Introduction}

Gastrulation is a critical developmental process required for germ layer formation and the establishment of the body plan (Arnold and Robertson, 2009). Gastrulation initiates with the emergence of the primitive streak in the proximal posterior epiblast. As the streak extends to the distal tip of the embryo, epiblast cells undergo an epithelial mesenchymal transition (EMT) to form the mesoderm layer between the epiblast and the visceral endoderm (VE) (Kinder et al., 1999; Lawson, 1999; Lawson et al., 1991). Epiblast cells ingressing through the anterior region of the elongating primitive streak intercalate into the VE to form the definitive endoderm layer, which will give rise to the gut tube, and subsequently, the epithelium of endodermal organs, such as the pancreas and intestine (Kwon et al., 2008; Lawson et al., 1986; Lawson and Pedersen, 1987). Gastrulation requires tight spatiotemporal coordination of embryo size, tissue migration and cell fate determination. Despite extensive research on these topics, it has been challenging to untangle the complex interplay among these three key components. Previous studies used embryological methods in pre-implantation embryos to investigate size regulation during the pre- and early-gastrulation stages. These experiments found that double-sized embryos, formed by aggregating two 8-cell stage morula, underwent size regulation before gastrulation. The double-sized embryos showed an increase in cell-cycle length compared to controls; in addition, they lacked the proliferative burst that normally occurs before gastrulation. These two modes of regulating cell proliferation allowed the aggregated embryos to reach a normal size and cell number before E7.0 (Buehr and McLaren, 1974; Lewis and Rossant, 1982) and then to gastrulate. Conversely, undersized mouse embryos generated by removing one or two blastomeres from the 4-cell stage preimplantation embryo, sustained a prolonged proliferative burst, leading to an increase in cell number before the initiation of gastrulation (Power and Tam, 1993). Another study on size regulation in the mammalian embryo examined the response to reduced cell number in the early post-implantation embryo, an experimentally more refractory stage. Following treatment with mitomycin to inhibit cell proliferation, E7.0 embryos, with $\sim 80 \%$ of their cells eliminated, could still recover and complete gastrulation (Snow and Tam, 1979). These elegant studies suggest that intrinsic mechanisms operate within the pre- and early post-implantation embryo to monitor and control cell number both before and at the onset of gastrulation, supporting regulative development as an important feature of early mammalian embryogenesis. However, standard embryological and pharmacological methods are inadequate to further investigate how altered cell number in the gastrulating embryo impacts tissue patterning and morphogenesis. Therefore, to ask whether mechanisms of regulative 
development continue to act during gastrulation, it is crucial to explore alternative approaches to perturbing cell number.

Although many genes encoding cell cycle related proteins have been genetically inactivated to explore the effects of cell proliferation on embryo size and morphogenesis, the resulting phenotypes are generally not suitable for studies of gastrulation. For example, Cyclin A2 (Ccna2) homozygous null mutants can be recovered only up to E5.5 (Murphy et al., 1997), whereas embryos lacking all D-type cyclins survive past gastrulation, with no overt phenotypes (Kozar et al., 2004). Another target for genetic perturbation of cell proliferation are the polymerases that replicate DNA. DNA Polymerase Delta (Pol $\delta$ ), the subject of this report, plays multiple critical roles in DNA replication, with functions in DNA synthesis and repair (Jain et al., 2018). In mammalian cells, the Pol $\delta$ contains 4 subunits: the catalytic subunit, p125 (Pold1), and three regulatory subunits, p50 (Pold2), p66 (Pold3) and p12 (Pold4). Pold1 consists of two functional domains: a N-terminal 3'-5' exonuclease with DNA proofreading activities and a Cterminal DNA polymerase that catalyzes DNA synthesis. Several mutant alleles have been generated for Pold1, but similar to targeted cell-cycle related genes mentioned above, the homozygous mutants either fail to survive beyond the onset of gastrulation or show no phenotypic defects during gastrulation. Null mutations in Pold1 cause peri-implantation lethality (Uchimura et al., 2009). Two missense mutations have been reported for Pold1: a D400A substitution in the exonuclease domain and a L604K substitution in the DNA polymerase activity site (Fig. S1A) (Uchimura et al., 2009; Venkatesan et al., 2007). While developmentally normal, Pold1 $1^{\text {D400A/D400A }}$ mice frequently died with swollen thymuses 3 months after birth (Uchimura et al., 2009). Pold $1^{+/ L 604 K}$ heterozygous mice underwent normal development but had a reduced lifespan and developed multiple tumor types, including lymphoma, adenoma and carcinomas of 100 the liver and lung (Venkatesan et al., 2007). Although no specific phenotypes were reported for Pold $1^{\text {L604K/L604K }}$ embryos, notably they died around E8.5, suggesting that missense mutations in the polymerase domain might perturb cell proliferation at a level compatible for investigation of gastrulation phenotypes.

In this study, we report a Pold1 hypomorphic mutation identified in a phenotype-based genetic screen for recessive mutations causing gastrulation defects in mouse embryos. This mutation altered a conserved residue (D939Y) in the Pold1 DNA polymerase domain, caused reduced Pold1 protein expression, and resulted in compromised DNA synthesis. Mutant embryos could be retrieved up to E8.5; at this stage they were small with a siren-like morphology; hence we 
named the mutant tiny siren (tyrn). We investigated embryo growth and cell lineage differentiation in tyrn mutants at developmental stages between E6.5 and E8.5. The tyrn mutation impaired cell proliferation without affecting anterior-posterior patterning, but severely disrupted tissue morphogenesis during gastrulation. Our findings suggest normal cell proliferation is essential for mesoderm lineage allocation and is required to coordinate embryo size with cell movement for proper morphogenesis.

\section{Results}

\section{tyrn mutant embryos show abnormal morphology but proper anterior-posterior} patterning

To study the genetic regulation of gastrulation, we performed mouse ENU mutagenesis screens to uncover mutant embryos with abnormal morphology at E8.5 (Garcia-Garcia et al., 2005; Hernandez-Martinez et al., 2019; Huangfu et al., 2003; Migeotte et al., 2011). We isolated a mutant (later named tyrn) that exhibited not only a smaller overall size, but also a striking body shape and orientation (Fig. 1A) that was distinctly from other mutant phenotypes we had observed at this stage (Bazzi et al., 2017; Garcia-Garcia et al., 2005; Hernandez-Martinez et al., 2019; Huangfu et al., 2003; Migeotte et al., 2011; Zhou and Anderson, 2010). Instead of forming a U-shaped embryo with a well extended anterior-posterior (A-P) axis, the tyrn mutant embryos had a short A-P axis and lay relatively flat along the posterior side of the yolk sac, with the head misoriented towards the distal tip (Fig. 1A). To determine if the irregularly shaped mutants had established and correctly positioned the A-P axis, we performed whole mount in situ hybridizations (WISH) at E8.5 to a diagnostic set of markers. We found that although tyrn mutant embryos did not form a well-structured head, they did express Foxg1 and Otx2, markers of forebrain and forebrain + midbrain, respectively, in discrete overlapping regions (Fig 1B-C). In addition, WISH detected relatively normal expression of $T$ (Brachyury), which marks the posterior tail bud and notochord (Fig. 1D), and of Foxa2, which labels the floor plate of the neural tube, posterior notochord, notochordal plate and gut endoderm (Fig. 1E). Therefore, despite causing a highly unusual body shape, the tyrn mutation does not affect A-P patterning.

\section{tyrn is a hypomorphic allele of Pold1}

140 To identify the causal mutation for the tyrn phenotype, we collected both wildtype and mutant embryos at E8.5 and performed whole-exome sequencing (Jain et al., 2017). We found a G to T transversion at nucleotide position 2815 of the Pold1 open reading frame that generated an aspartate to tyrosine substitution (D939Y) within the DNA polymerase domain (Fig. 2A). The 
mutated aspartate residue is highly conserved across eukaryotic organisms from budding yeast to humans (Fig. 2B). Western blot analysis showed a reduced level of Pold1 in tyrn mutant embryos (Fig. 2C). To ask whether Pold1 is the causative gene underlying the tyrn mutant phenotype, we performed a complementation test using the Pold $1^{\text {tm } 1 b}$ null allele, derived from embryonic stem cells carrying a "knockout-first" tm1a allele (Skarnes et al., 2011) (Fig. 2D, Fig S1B). No Pold $1^{\text {tm1b/tm1b }}$ embryos were recovered at post-implantation stages, consistent with the phenotype previously reported for Pold1 null mutants (Uchimura et al., 2009). The Pold1yrn/tm1b embryos produced from a Pold $1^{\text {tm } 1 b /+}$ and tyrn/+ cross did not initiate gastrulation and failed to survive past $\sim$ E7.5 (Fig. 2D), demonstrating that the $\operatorname{tm} 1 \mathrm{~b}$ and tyrn mutations failed to complement. Moreover, the phenotype displayed by Pold $1^{t y r n / t m 1 b}$ embryos was more severe

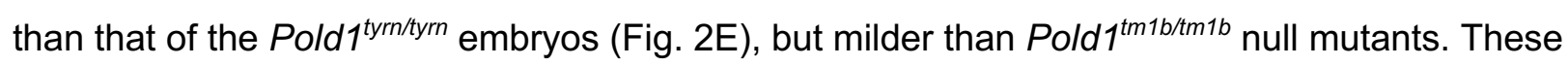

155 findings indicate that Pold $1^{\text {tyrn }}$ is a hypomorphic allele and that perturbation of Pold 1 function is responsible for the phenotypes observed in tyrn mutants.

\section{The tyrn mutation impairs DNA synthesis and cell proliferation}

Based on the published cryo-electron microscopy structure of the human Pol $\delta$ (Lancey et al., 2020), the aspartate residue mutated in tyrn embryos resides in the thumb domain of the Cterminal DNA polymerase domain of POLD1 (Fig. 2A, Fig. S2A-C). Because of the important role of the thumb domain in stabilizing Pol $\delta$ at the primer-template junction during DNA synthesis (Jain et al., 2018), we hypothesized that the tyrn mutation may impair and reduce the DNA synthesis activity of Pol $\delta$. We used a primer extension assay and mouse EdU labeling to test Pol $\delta$ polymerase activity in vitro and in vivo, respectively. For the primer extension assay, we modeled the Pold1 D939Y missense mutation in budding yeast Pol $\delta$ by introducing the equivalent mutation, D941Y, in the catalytic subunit, Pol3 (Devbhandari and Remus, 2020) (Fig. $3 \mathrm{~A}$ ). We purified wildtype Pol $\delta$ or Pol $\delta^{\text {D941Y }}$ (with Pol3 ${ }^{\mathrm{D} 941 Y}$ ) after overexpression in budding yeast (Fig. 3B). Analysis of the DNA synthesis products by denaturing gel-analysis reveals that

170 both overall DNA synthesis and the level of full-length DNA products were significantly reduced in the presence of Pol3 ${ }^{\mathrm{D} 941 Y}$ when compared to the wildtype Pol $\delta$ (Fig. 3C-E). These data demonstrate that the D941Y substitution in Pol3, and by extension the corresponding D939Y substitution in Pold1, impairs the DNA polymerase activity of Pol $\delta$, possibly by decreasing its processivity. 
To test if DNA synthesis is affected in tyrn embryos, we performed in vivo EdU labeling at E6.0, E6.5 and E7.0, the window of time during which the size difference between wildtype and mutant embryos emerges. We observed a significant reduction in EdU incorporation in mutants compared to wild types at all stages (Fig. 4A-B). Both wildtype and mutant embryos showed a substantial increase in cell number from E6.0 to E7.0, but tyrn embryos exhibited a slower growth rate starting from E6.5; by E7.0 mutant embryos had significantly lower numbers of cells compared to wildtype embryos (Fig. 4C). The reduction in cell number is not due to increased cell death; based on cleaved Caspase-3 staining, levels of cell apoptosis were similar between wildtype and mutant embryos during this period (Fig. S3). However, mutant embryos showed

185 increased cell apoptosis at around E7.5, with apoptotic cells concentrated at the distal tip, the prospective location of the abnormal small head at E8.5 (Fig. 4D). Taken together, we conclude that the D939Y missense mutation impairs Pold1 polymerase activity, which, together with the reduced Pold1 protein expression in tyrn embryos, impedes cell proliferation and leads to a reduction in embryo size during gastrulation.

\section{The tyrn mutation does not affect anterior visceral endoderm (AVE) positioning}

The mispositioned head, and the abnormal morphology of E8.5 tyrn mutants, indicated that the A-P axis, although established, was shifted in orientation along the proximal-distal axis. Proper formation of the A-P axis depends on the anterior migration of a morphologically distinct population of VE cells from their position at the distal tip of the E5.5 embryo (Rivera-Perez et al., 2003); by E5.75-E6.0 this cell population will reach the anterior epiblast - extraembryonic ectoderm boundary and form the AVE (anterior visceral endoderm) (Srinivas et al., 2004). Multiple studies have shown that defects in AVE migration can lead to abnormal positioning of the A-P axis and mislocalized head phenotypes (Acampora et al., 2009; Clements et al., 2011; Kojima et al., 2014; Lu et al., 2001; Martinez-Barbera et al., 2000; Rossant and Tam, 2009). Therefore, we asked whether the AVE resided in its normal anterior position in tyrn mutants at E6.5. Although the tyrn mutants tended to be smaller at this stage, they were morphologically indistinguishable from wildtype littermates. WISH showed that tyrn mutants expressed two archetypal AVE markers, Dkk1 (a Wnt antagonist) and Cerl (a Nodal antagonist), in a pattern comparable to that of wildtype embryos (Fig. 5A-B). We validated these findings by crossing in the Hhex-GFP transgene reporter to fluorescently label AVE cells in tyrn mutants (Rodriguez et al., 2001). Consistent with the Dkk1 and Cer1 in situ hybridization results, we detected the Hhex-GFP expressing AVE cells at the anterior boundary between the embryonic and 
extraembryonic regions in tyrn embryos at E6.5 (Fig. 5C). These results indicate that AVE migration and A-P axis establishment proceed normally in pre-gastrulation stage tyrn mutants.

\section{The tyrn mutation affects primitive streak extension and head position at E7.5}

At E6.5, the AVE resided at the expected location in tyrn mutants; yet the position of the tyrn embryo's anterior region was shifted toward the distal tip at E7.5. We examined the expression of multiple anterior-specific markers to visualize the organization of the anterior region in both wildtype and mutant embryos at E7.5. As depicted in Fig. 6A-B, wildtype embryos expressed Sox2 uniformly throughout the anterior epiblast, whereas the tyrn mutants expressed Sox 2 in a discontinuous pattern, with more intense staining in the distal epiblast than in the proximal/anterior region. In the wildtype E7.5 embryo in the left panel of Fig. $6 C$ \& D, the HhexGFP transgene labels anterior definitive endoderm (ADE) as well as remaining AVE cells. In contrast, the Hhex-GFP staining resides predominantly in distal region of the E7.5 tyrn mutant, with no obvious A-P asymmetry (Fig. 6C-D, right panels). Similarly, cells expressing Otx2, a head organizer marker, lie distally in mutant embryos compared to their anterior position in wild types (Fig. 6E).

The AVE serves as a transient anterior signaling center at $\sim \mathrm{E} 6.5$. As gastrulation progresses, AVE cells disperse into the extraembryonic/embryonic boundary and the anterior portion of the extraembryonic yolk sac (Lawson and Pedersen, 1987; Rivera-Perez et al., 2003; Shimono and Behringer, 2003; Tam et al., 2007). Meanwhile, the ADE and axial mesoderm (AME), both of which strongly express Nodal and Wnt antagonists (Arnold and Robertson, 2009), gradually migrate anteriorly to replace the AVE and become new sources of anterior signaling. We hypothesized that the abnormal orientation of the A-P axis might reflect distal positioning of AME and ADE. Fig. 6A, D, F examines the expression of Foxa2, a marker of AME and ADE, in wildtype and tyrn embryos at E7.5. Emerging Foxa2-labeled ADE and AME cells reside in the distal anterior region in wild types, but these cells sit more posteriorly in mutants.

The aberrant posterior positioning of $A D E$ and $A M E$, both derivatives of anterior primitive streak (APS) (Arnold and Robertson, 2009; Lawson, 1999; Lawson et al., 1991), suggested that primitive streak extension was defective in tyrn mutants. To compare the organization of the 240 primitive between wildtype and mutant embryos, we performed immunofluorescence for $\mathrm{T}$ (Brachyury), a marker of nascent mesoderm emanating from the primitive streak, including AME (Fig. 6B-C). In E7.5 wildtype embryos, T staining showed that the primitive streak had extended 
to the distal tip and generated AME derivatives of the anterior primitive streak. In contrast, T staining of E7.5 tyrn embryos indicated that the primitive streak had extended only to the midpoint of the posterior side (Fig. 6B-C). Also pointing to impaired primitive streak elongation in tyrn embryos, WISH detected irregularities in the production and positioning of paraxial and extraembryonic mesoderm. Whereas expression of $T b x 6$, a marker of paraxial mesoderm, was found along the posterior side of the wildtype embryo, down to the distal tip, it was restricted to the posterior-proximal region in tyrn, consistent with a short primitive streak (Fig. 6G). Twist expression labels two distinct mesodermal populations at E7.5; anterior mesoderm precursors to cranial mesenchyme and extraembryonic mesoderm of the allantois (Bildsoe et al., 2009). Of note, the E7.5 tyrn mutant generated Twist expressing anterior mesoderm but lacked Twist expressing cells of the allantois (Fig. $6 \mathrm{H}$ ). The mutant embryos also expressed greatly reduced levels of $T b x 4$, another marker of allantoic mesoderm (Fig. 6I). These findings indicate that tyrn mutants have very few extraembryonic mesoderm cells inside the allantois. Taken together, these results suggest that the impaired extension of the primitive streak in tyrn embryos causes defects in distribution of multiple mesoderm derivatives, including the distal positioning of the AME and ADE at E7.5. Mutant embryos continued with head morphogenesis and eventually formed a head at the distal tip with abnormal morphology.

\section{Discussion}

The regulation of cell number plays an integral role in mouse embryo size determination during the pre-gastrulation stages. Earlier studies used embryo manipulation or pharmacological methods to alter cell numbers in pre- and early post-implantation mouse embryos. The findings showed, that prior to gastrulation, mouse embryos have the ability to sense and respond to an acute, global alteration in cell numbers and achieve normal size. Thus, robust size regulatory mechanisms must operate intrinsically in the mouse embryo to ensure proper size before the onset of gastrulation (Lewis and Rossant, 1982; Power and Tam, 1993). These classic studies raise many questions about when, in what cell populations, and how size regulatory mechanism function; questions that are not amenable to approaches involving the global loss or gain of cells especially for investigations around and after gastrulation. Fine-tuned genetic methods could allow manipulation of candidate components of the size regulatory process for extended period. Here, we assessed the impact on embryo growth and morphogenesis of a hypomorphic mutation that reduces levels of DNA proliferation at gastrulation stages. The tyrn mutants from

275 our ENU mutagenesis screen provided an excellent time window for us to investigate the roles of embryo growth in gastrulation. The tyrn mutation disrupted the polymerase function of Pold1 
and caused reduced cell proliferation in vivo. Mutant embryos did not merely exhibit a wildtypelike morphology with a proportionally reduced size, or a random shape with no underlying logic. Instead, it showed a siren-like morphology, with the head located closer to the distal tip rather than residing at the normal anterior region seen in the wild types. Further phenotypic analyses from E6.5-E7.5 stages revealed that the mispositioned head as well as the general abnormal morphology seen in tyrn embryos likely resulted from the defective primitive streak extension during gastrulation. The short primitive streak led to the distal positioning of the AME and ADE, which caused the orientation of the anterior-posterior axis to be shifted towards the distalproximal axis (Fig. 7). The tyrn mutants showed remarkable impact of reduced cell proliferation during gastrulation. The impaired DNA synthesis machinery caused prolonged defects in cell proliferation, leading to a discoordination of embryo growth with lineage specification and tissue morphogenesis. Such discoordination resulted in embryos with abnormal morphology and incorrect orientation inside the decidua. These results indicate that the embryo growth needs to be highly coordinated with lineage specification and tissue morphogenesis for normal gastrulation.

Our study suggests that mesoderm lineage allocation during gastrulation is affected by the global reduction of cell proliferation. Primitive streak is initially induced at the posterior-proximal pole where high levels of BMP, FGF, Wnt and Nodal signals present (Brennan et al., 2001; Ciruna and Rossant, 2001; Conlon et al., 1994; Huelsken et al., 2000; Mishina et al., 1995). The primitive streak elongates and extends to the distal tip as gastrulation progresses. The cell fate of mesoderm derivatives is determined by timing and positions of their ingression through the streak (Vincent et al., 2003; Winnier et al., 1995). The normal cell proliferation rate guarantees coordination of embryo size expansion and the signal gradient, enabling cells along the streak being exposed to different signal combination and contribute to different mesoderm subtypes. Such coordination was disrupted due to reduced cell proliferation: the primitive streak failed to fully extend, which could lead to aberrant signal gradients along the streak. We found that axial mesoderm, paraxial mesoderm subtypes were generated, albeit located more posteriorly. The 305 greatly reduced allantoic mesoderm may be a consequence of altered posterior signal environment. It could also reflect a general developmental delay: the cell differentiation along the streak follows the anterior-posterior order and the allantoic mesoderm arises relatively late during gastrulation. Another possibility is that these extraembryonic mesodermal cells may originate from a highly proliferative cell population (Snow and Tam, 1979) that could be more 
sensitive to inhibition of DNA synthesis. These questions may be addressed in future through the perturbation of embryonic cell numbers in specific cell populations during gastrulation.

Cell number regulation occurs throughout different stages of embryonic development. The effects of cell number perturbation on tissue/organ size and morphogenesis can vary greatly based on developmental stages and tissue/organ types. The mandibular hypoplasia, deafness, progeroid and lipodystrophy (MDPL) syndrome, a multisystem disorder, has been associated with heterozygous in-frame deletion of Ser605 that causes loss of POLD1 activity (Elouej et al., 2017; Fiorillo et al., 2018; Sasaki et al., 2018; Weedon et al., 2013). The reported patients presented growth retardation, sensorineural deafness, loss of subcutaneous adipose tissue and insulin resistance, indicating that decreased POLD1 activity exerts organ specific effects. Other studies have also identified organ-specific responses to loss of tissue-specific progenitors. Removing the embryonic limb field in amphibians or chickens has no effect on the final limb size as the limb is capable of robust compensatory proliferation (Holder, 1981; Summerbell, 1981), and similar compensatory growth has been observed for the developing liver progenitors (Bort et al., 2006). In contrast, the final pancreas size is determined by the initial pancreatic progenitor pool and there is a lack of significant compensatory growth (Stanger et al., 2007). These studies demonstrate the importance of studying cell number regulation in diverse tissue and organ types at different stages of embryonic development. Our findings underscore the power of unbiased phenotype-based forward genetic screening approaches and highlight the value of using ENU-induced hypomorphic alleles to uncover new biological functions of well characterized genes such as Pold1. For decades, embryo size regulation has become the "forgotten classics" in the developmental biology field (https://thenode.biologists.com/forgottenclassics-regulating-size-mouse-embryo/research/). In future studies, one may use carefully chosen genetic models coupled with modern developmental biology techniques such as live imaging and single cell genomics to revisit this important topic in diverse developmental contexts beyond the early stages of embryogenesis. 


\section{Materials and Methods \\ Mouse Strains (Mus musculus)}

The parental JM8.N4 mouse ES Cell Strain (strain origin: C57BL/6N, male, black coat, nonagouti, MGI ID: 4431772) (Skarnes et al., 2011) carrying one Pold1 $1^{\text {tm1a(EUCOMM)Wtsi }}$ (Pold1 $^{\text {tm1a }}$ ) allele was imported from European Mouse Mutant Repository (EUMMCR) unit. The selected Pold1-G09 ES cell clone passed the karyotype check performed by Molecular Cytogenetics Core Facility, Memorial Sloan Kettering Cancer Center (MSKCC) and was free of mouse pathogens tested by MSKCC Center of Comparative Medicine \& Pathology. Pold1-G09 ES cell clone was injected into the female B6(Cg)-Tyr $\mathrm{C}^{\mathrm{c}-2 \mathrm{~J}} / \mathrm{J}$ (albino C57BL/6J, or B6-albino, non-agouti, The Jackson Laboratory) donor mice by MSKCC Mouse Genetics Core Facility. 14 out of the 19 pups alive were chimera. Male chimeras were crossed with albino FVB/NJ (FVB, containing homozygous dominant agouti locus $A / A$ ) females to generate heterozygous offspring carrying the Pold $1^{\text {tm1a }}$ allele. Germline transmission was determined by the presence of agouti pups and genotyping. The Pold $1^{\text {tm1a }}$ allele contains a lacZ cassette for genotyping. Mice carrying Pold $1^{\text {tm } 1 b}$ (null) allele were generated by crossing Pold $1^{\text {tm1a/+ }}$ mice with CAG-Cre transgenic mice (The Jackson Laboratory) to remove the critical exons between exon 3 to exon 10 . The lacZ cassette remained in Pold $1^{\text {tm1b }}$ allele and was used for genotyping. The Hhex-GFP strain was a gift from Anna Katarina Hadjatonakis (Rodriguez et al., 2001). Mice that were 8-16 weeks old were used to generate E6.5 to E8.5 embryos. Analysis of the mutant phenotype was performed in the FVB genetic background or FVB-B6 mixed background. Mice were housed and bred under standard conditions in accordance with Institutional Animal Care and Use Committee (IACUC) guidelines. The MSKCC IACUC approved the experiments.

\section{ENU Allele Isolation, Sequencing and Genotyping.}

The tyrn allele was generated by mouse ENU mutagenesis screens using C57BL/6J males and identified based on its embryonic phenotype at E8.5, as previously described (Garcia-Garcia et al., 2005). To obtain genomic DNA, E8.5 mutant embryos were pooled into 3 sample groups and snap frozen on dry ice. Gentra Puregene kit (QIAGEN) was used for genomic DNA extraction of mutant embryos. Whole-exome sequencing was performed at the MSKCC Integrated Genomics Operation. Exome capture was performed using SureSelectXT kit (Agilent Technologies) and SureSelect Mouse All Exon baits (Agilent Technologies). An average of 100 million 75-bp paired reads were generated. Sequencing data analysis was performed using the methods described in (Jain et al., 2017). To obtain a list of potential phenotype-causing lesions, variants were filtered further to only include those that 1) were not found in dbSNP, 2) were not 
found in samples from other lines sent for this sequencing batch, 3) were shared among all 3 mutant samples, 4) were with coverage (sequencing read depth) of at least 6,5 ) were homozygous, 6) were exonic only. 11 variants were found in 8 genes/annotations, 6 nonsynonymous, of which Pold1 was one: Pold1: NM_011131: exon23: c. G2815T: p.D939Y. After examining the known phenotypes of published alleles or functional annotations of these candidate genes, we re-examined non-exonic and heterozygous mutations and eliminate read depth threshold to explore other possibilities. After going through the whole dataset (developed by Devanshi Jain from Scott Keeney lab)(Jain et al., 2017) containing all our exome sequencing submissions over the years to catalog universal variants we could eliminate, plus additional analysis with the above criteria, we confirmed that the sole candidate variant was in Pold1. The tyrn allele has a single $\mathrm{G}$ to $\mathrm{T}$ transversion in the 2815 nucleotide position of exon 23 in Pold1 coding sequence (G2815T), causing a missense mutation (in the amino acid position 939 aspartic acid to tyrosine, D939Y). The G2815T mutation created a Rsal restriction site used for genotyping.

\section{Complementation Test}

Pold $1^{+/ t m 1 b}$ females were crossed with tyrn/+ males to produce embryos. Embryos at E7.5 and E8.5 stages were retrieved and grouped into wild types and mutants based on phenotypes. No mutant embryos were found at E8.5 stage. The genotypes of E7.5 embryos matched their phenotypes: Embryos with mutant phenotype were Pold $1^{\text {tyrn/tm } 1 b} ;$ Pold $1^{+/+}$, Pold $1^{+/ t y r n}$ and Pold $1^{+/ t m 1 b}$ showed wildtype phenotypes.

\section{Embryo Harvesting and Dissections}

Pregnant FVB female mice bearing embryos at E6.5-10.5 stages were euthanized by cervical dislocation. Uterus were taken out for dissection based on approved mouse protocol. Embryos were dissected from decidua inside uterus in cold 0.4\% Bovine Serum Albumin (Sigma-Aldrich) in Phosphate-buffered Saline (PBS) using fine forceps and Leica dissection scope. Embryos were fixed in 4\% paraformaldehyde (PFA) on ice for at least 4 hours and washed with PBS for 5 minutes $X 3$ times at room temperature (RT). Fixed embryos were stored in PBS at $4^{\circ} \mathrm{C}$ for 400 future experiments.

\section{In Situ Hybridization}

Fixed embryos were sequentially dehydrated in $25,50 \%, 75 \%$ methanol-depc PBS and $100 \%$ methanol and stored in $-20^{\circ} \mathrm{C}$. Embryos were taken out from methanol and sequentially 
rehydrated in 75\%, 50\%, 25\% methanol-depc PBS before experiments. In situ Hybridization were performed following standard protocol (Eggenschwiler and Anderson, 2000). Briefly, after rehydration, embryos were incubated in $1 \mu \mathrm{g} / \mathrm{mL}$ Proteinase K/PBS solution for 3 to 7 min and hybridized in hybridization solution with RNA probes at $70^{\circ} \mathrm{C}$ overnight. A series of washes in $2 \mathrm{X}$ SSC and MAB solutions were performed the next day. The embryos were incubated in 1:10000 anti-Digoxigenin antibody (Roche) in blocking buffer (1\% blocking reagent [Roche], 10\% heatinactivated goat serum, 0.1\% Tween-20) in PBS overnight and washed extensively in PBS $+0.1 \%$ Tween-20. Embryos were incubated in BM purple solution (Roche) at RT protected from light until the purple color was developed.

\section{EdU labeling}

EdU (5-ethynyl-2'-deoxyuridine) powder (Invitrogen) was dissolved in sterile PBS into a working concentration of $2.5 \mathrm{mg} / \mathrm{ml}$. Mice were weighed and injected with EdU solution $(25 \mathrm{mg} / \mathrm{kg})$ intraperitoneally. Embryos were harvested 2 hours after injection and fixed in 4\% PFA overnight. Fixed embryos were washed with PBS $+3 \%$ BSA twice and then permeabilized in PBS $+0.5 \%$ Triton X-100 at RT for 20 minutes. Embryos were washed in PBS+3\% BSA after permeabilization. Embryos were incubated with reaction cocktail made from Click-iT ${ }^{\mathrm{TM}}$ EdU Cell Proliferation Kit for Imaging, Alexa Fluor 637 dye kit (Invitrogen) at RT for 30 minutes, protected from light. Cocktail was removed after incubation and embryos were washed in PBS $+3 \%$ BSA twice.

\section{Immunofluorescence and Confocal Microscopy}

Fixed embryos were store in PBS at $4^{\circ} \mathrm{C}$ before use. Embryos for cryosection were dehydrated in $30 \%$ Sucrose-PBS at $4^{\circ} \mathrm{C}$ overnight. Embryos were embedded in Tissue-Tek ${ }^{\circledR}$ O.C.T Compound (Sakura Finetek) and frozen on smashed dry ice immediately after embedding.

430 Embedded embryos were stored in $-80^{\circ} \mathrm{C}$ before use. Embryos were sectioned in $10 \mu \mathrm{m}$ using Leica CM1520 Cryostat. Section slides were stored in $-80^{\circ} \mathrm{C}$ before use. For whole-mount staining embryos, embryos were permeabilized with PBS $+0.5 \%$ Triton X-100 for 1 hour at RT. For section staining, slides were dried for 30 minutes and incubated in blocking buffer $(0.1 \%$ TritonX-100, 1\% heat-inactivated donkey serum [Gemini: Bio-produces] in PBS) for 1 hour at 
Embryos and slides were incubated with primary antibodies at $4^{\circ} \mathrm{C}$ overnight on rotator. Embryos and slides were washed with PBS for 10 minutes $X 3$ times the next day. Section slides or embryos were incubated in blocking buffer containing specific secondary antibodies (Invitrogen,1:500) and DAPI (1:1000) for 2 hours at RT. For whole mount staining embryos, embryos were washed in PBS for 5 minutes $X 3$ times and incubated in FocusClear (CelExplorer.Co) for 20 minutes at RT, protected from light. Embryos were mounted with MountClear (CelExplorer.Co) and stored at $4^{\circ} \mathrm{C}$. For cryosection staining, slides were washed in PBS for 5 minutes X 3 times and mounted with ProLong Gold Antifade Mountant (Thermo Fisher Scientific) and stored at $4^{\circ} \mathrm{C}$. Both embryos and sections were imaged using Leica SP8 inverted laser scanning confocal microscope. Confocal images were reconstructed using Fiji (ImageJ) open-source image processing software.

\section{Immunoblotting}

E8.5 wildtype and mutant embryos were harvested and pooled separately and stored in $-80^{\circ} \mathrm{C}$ before experiments. Tissues were homogenized in cold lysis buffer $(0.1 \%$ NP-40, 50mM Tris$\mathrm{HCl}$ [pH 7.2], 250mM NaCl, 2mM EDTA, phosphatase inhibitor mixture I and II [Calbiochem] and one tablet of Minicomplete [Roche] per 10ml) on ice. Lysate was left on shaker at $4^{\circ} \mathrm{C}$ for another 30 minutes. Lysate was centrifuged at maximum speed (12000 rpm) to collect supernatant. Protein concentration was determined through BSA-based protein assay using Quick Start Bradford 1X Dye Reagent (BIORAD) and adjusted to 2ug/ul. Samples were mixed $1: 1$ with $2 X$ SDS loading buffer and denatured at $95^{\circ} \mathrm{C}$ for 5 minutes. Samples were loaded with equal amount onto 8\% SDS-PAGE gels and ran for 2 hours under 150V in 1X SDS at RT. Proteins were transferred to PVDF membranes under $15 \mathrm{~V}$ overnight at $4^{\circ} \mathrm{C}$. Membranes were incubated in blocking buffer containing (TBST+5\% BSA) for 1hour at RT and incubated with primary antibody in blocking buffer at $4^{\circ} \mathrm{C}$ overnight: POLD1 (Abcam Cat\# ab168827,1:500); GAPDH (Santa Cruz Biotechnology Cat\# sc-32233, RRID:AB_627679, 1:1000). Membranes were washed with TBST and incubated with specific secondary antibodies for 1 hour at RT. Membranes were washed with TBST and incubated with Pierce ECL Western Blotting Substrate (Thermo Fisher Scientific) for 5 minutes and were film-exposed to show target bands.

\section{Primer Extension Assay}

470 Primer extension reactions were performed at $30^{\circ} \mathrm{C}$ in polymerization buffer $(25 \mathrm{mM} \mathrm{Tris}-\mathrm{HCl} \mathrm{pH}$ 7.5, $8 \mathrm{mM}$ Magnesium Acetate, $5 \mathrm{mM}$ Potassium Glutamate, 5\% Glycerol). Purified proteins used in the primer extension assays were purified as previously described (Devbhandari and 
Remus, 2020). DNA template for the assay was generated by annealing a primer (5'CCCAGTCACGACGTTGTAAAACG-3') to M13mp18 single-stranded DNA (New England

Biolabs, N4040S). The assay was initiated by incubation of $1 \mathrm{nM}$ of DNA template with $1 \mathrm{mM}$ ATP, 1 mM DTT, $80 \mu \mathrm{M}$ dATP, $80 \mu \mathrm{M}$ dGTP, $80 \mu \mathrm{M}$ dCTP and $400 \mathrm{nM}$ of RPA for 5 minutes. PCNA and RFC were then added to $70 \mathrm{nM}$ and $4 \mathrm{nM}$, respectively, and incubation was continued for 5 minutes. Then, $33 \mathrm{nM}$ of $\alpha-{ }^{32} \mathrm{P}-\mathrm{dATP}(3,000 \mathrm{Ci}$ per mmol) and $4 \mathrm{nM}$ of either Pol $\delta^{\mathrm{WT}}$ or Pol $\delta^{\mathrm{D} 941 \mathrm{Y}}$ was added to the reaction resulting in a primer extension by 9 base pairs (due to lack of dTTP). After $5 \mathrm{~min}, 80 \mu \mathrm{M}$ dTTP were added to the mix for synchronous primer extension. Equal volume aliquots of this reaction $(18 \mu \mathrm{l})$ were removed from the master reaction $(100 \mu \mathrm{l})$ at indicated times and stopped by adding EDTA and SDS to final concentrations of $40 \mathrm{mM}$ and $0.25 \%$, respectively. Products were fractionated on a $0.8 \%$ alkaline agarose gel (30 mM NaOH and 2 mM EDTA), dried and imaged using Typhoon FLA 7000. Quantification of the gel images was performed using the ImageJ.

\section{Quantitation of Total Cells, EdU Signal Intensity, EdU-Positive Cells}

E6.0, E6.5 and E7.0 whole mount EdU-labeled embryos were imaged using Leica SP8 inverted laser scanning confocal microscope and confocal z stacks of embryos were generated. Mutant and wildtype embryos of the same stage were imaged under the same conditions. For each stage, 3 embryos per genotype were imaged for quantification. Optical sections of each embryo were imported into Imaris (version 9.5, Oxford Instruments), and 3D-reconstitution along Z-axis was performed for data analysis. We used spots function in Imaris to automatically segment all DAPI-positive cells (both embryonic and extraembryonic tissues) to obtain a total cell count for each embryo. EdU signal intensity of each segmented cell was automatically measured and the background signal was subtracted. Cells with EdU signal above 10 arbitrary unit were considered EdU positive and counted as EdU positive cells by the software. We used Prism 9 (GraphPad) to perform normalization of EdU signal intensity. At each stage, the EdU signal of each EdU positive cell was normalized to the maximum EdU signal intensity. Data points were presented as box plot to show the distribution of EdU signal intensity among EdU positive cell population. Within box plot, the median is represented by the horizontal dividing line and the top and bottom of the box represents the seventy-fifth and twenty-fifth percentile, with the whiskers indicating the maximum and minimum points. Two-tailed Student's $t$ test was performed to evaluate the significance of all measurements. 


\section{Statistics and Graphs}

Embryo images $\mathrm{n}=3$ for all the experiments. For primer extension assay, 3 biological replicates were performed. We used Prism 9 to perform 2-tailed Student's $t$ test to evaluate the significance of all measurements and generated box plots and non-linear fitted curves. For the

510 structure of human DNA polymerase $\delta$, the original structure was imported and processed in PyMOL (The PyMOL Molecular Graphics System, Version 1.2r3pre. Schrödinger, LLC)

\section{Data availability}

The structure of human DNA polymerase delta holoenzyme was pulled from protein data bank 515 (PDB). PDB DOI: 10.2210/pdb6TNY/pdb. EM Map EMD-10539: EMDB EMDataResource.

\section{Primer information}

\begin{tabular}{|l|l|l|}
\hline Target gene (allele) & Forward 5'-3' & Reverse 5'-3' \\
\hline Pold1 (tyrn) & atgcttctgacccctgcacc & gagtgtcgatgggcaggctg \\
\hline Pold1 (wildtype) & ggagttgctcctgtggaagacc & aggaatgaggtgaaccacatcccg \\
\hline Pold1 (tm1a or tm1b) & atcctctgcatggtcaggtc & cgtggcctgattcattcc \\
\hline CAG-Cre & attgctgtcacttggtcgtggc & ggaaaatgcttctgtccgttgc \\
\hline Hhex-GFP & aagttcatctgcaccaccg & tccttgaagaagatggtgcg \\
\hline
\end{tabular}




\section{Acknowledgments:}

The authors thank Edward Espinoza for Pold1 cDNA preparation and validation of Pold1 G2815T mutation, Dr. Devanshi Jain for sharing the methods of data analysis. The authors thank MSKCC Integrated Genome Operation for performing whole-exome sequencing and exome-wide SNP dataset, MSKCC Molecular Cytogenetics Core Facility for karyotype check, 525 MSKCC Center of Comparative Medicine and Pathology for pathogen exam, MSKCC Mouse Genetics Core Facility for ESC clone injection, MSKCC Molecular Cytology Core Facility (Dr. Boyko, Mr. Tipping, Mr. Feng) at for assistance with confocal imaging and image processing. The authors thank Dr. Anna-Katerina Hadjantonakis for providing Hhex-GFP mouse strain, Jonathan Pai from Hadjantonakis' lab for methods of clearing embryos. The authors thank Dr. Jennifer Zallen and Dr. Maria Jasin for helpful suggestions on this project.

\section{Author contributions}

T.C. and K.V.A. conceived the study and designed the experiments. T.C., D.H. and E.L. interpreted the data and wrote the manuscript with inputs from other authors. K.V.A. and H.A. performed the ENU mutagenesis screen. T.C. maintained mouse lines, performed animal crossing, genotyping, dissection, in situ hybridization, immunofluorescence staining, confocal imaging, cloning, immunoblotting and data analysis. H.A. performed genomic DNA preparation and whole-exome sequencing data analysis. D.R. supervised and S.D. performed in vitro primer extension assays and quantifications.

\section{Declaration of Interests}

The authors declared no competing interests.

\section{Funding}

545 This work was supported by grants from the National Institute of Health (NIH) (R01HD035455 to K.V.A., subsumed by Drs. Hadjantonakis, Joyner and Huangfu (D.H.) after the sudden death of Dr. Anderson; R01GM107239 to D.R.) and MSKCC Cancer Center Support Grant (P30CA008748). 
Acampora, D., Di Giovannantonio, L. G., Di Salvio, M., Mancuso, P. and Simeone, A. (2009). Selective inactivation of Otx2 mRNA isoforms reveals isoform-specific requirement for visceral endoderm anteriorization and head morphogenesis and highlights cell diversity in the visceral endoderm. Mech Dev 126, 882-897.

Arnold, S. J. and Robertson, E. J. (2009). Making a commitment: cell lineage allocation and axis patterning in the early mouse embryo. Nat Rev Mol Cell Biol 10, 91-103.

Bazzi, H., Soroka, E., Alcorn, H. L. and Anderson, K. V. (2017). STRIP1, a core component of STRIPAK complexes, is essential for normal mesoderm migration in the mouse embryo. Proc Natl Acad Sci U S A 114, E10928-E10936.

Bildsoe, H., Loebel, D. A., Jones, V. J., Chen, Y. T., Behringer, R. R. and Tam, P. P. (2009). Requirement for Twist1 in frontonasal and skull vault development in the mouse embryo. Dev Biol 331, 176-188.

Bort, R., Signore, M., Tremblay, K., Martinez Barbera, J. P. and Zaret, K. S. (2006). Hex homeobox gene controls the transition of the endoderm to a pseudostratified, cell emergent epithelium for liver bud development. Dev Biol 290, 44-56.

Brennan, J., Lu, C. C., Norris, D. P., Rodriguez, T. A., Beddington, R. S. and Robertson, E. J. (2001). Nodal signalling in the epiblast patterns the early mouse embryo. Nature 411, 965-969.

Buehr, M. and McLaren, A. (1974). Size regulation in chimaeric mouse embryos. J Embryol Exp Morphol 31, 229-234.

Ciruna, B. and Rossant, J. (2001). FGF signaling regulates mesoderm cell fate specification and morphogenetic movement at the primitive streak. Dev Cell 1, 37-49.

Clements, M., Pernaute, B., Vella, F. and Rodriguez, T. A. (2011). Crosstalk between Nodal/activin and MAPK p38 signaling is essential for anterior-posterior axis specification. Curr Biol 21, 1289-1295.

Conlon, F. L., Lyons, K. M., Takaesu, N., Barth, K. S., Kispert, A., Herrmann, B. and Robertson, E. J. (1994). A primary requirement for nodal in the formation and maintenance of the primitive streak in the mouse. Development 120, 1919-1928.

Devbhandari, S. and Remus, D. (2020). Rad53 limits CMG helicase uncoupling from DNA synthesis at replication forks. Nat Struct Mol Biol 27, 461-471.

Eggenschwiler, J. T. and Anderson, K. V. (2000). Dorsal and lateral fates in the mouse neural tube require the cell-autonomous activity of the open brain gene. Dev Biol 227, 648-660. 
Elouej, S., Beleza-Meireles, A., Caswell, R., Colclough, K., Ellard, S., Desvignes, J. P., Beroud, C., Levy, N., Mohammed, S. and De Sandre-Giovannoli, A. (2017). Exome sequencing reveals a de novo POLD1 mutation causing phenotypic variability in mandibular hypoplasia, deafness, progeroid features, and lipodystrophy syndrome (MDPL). Metabolism 71, 213-225.

Fiorillo, C., D'Apice, M. R., Trucco, F., Murdocca, M., Spitalieri, P., Assereto, S., Baratto, S., Morcaldi, G., Minetti, C., Sangiuolo, F., et al. (2018). Characterization of MDPL Fibroblasts Carrying the Recurrent p.Ser605del Mutation in POLD1 Gene. DNA Cell Biol.

Garcia-Garcia, M. J., Eggenschwiler, J. T., Caspary, T., Alcorn, H. L., Wyler, M. R., Huangfu, D., Rakeman, A. S., Lee, J. D., Feinberg, E. H., Timmer, J. R., et al. (2005). Analysis of mouse embryonic patterning and morphogenesis by forward genetics. Proc Natl Acad Sci U S A 102, 5913-5919.

Hernandez-Martinez, R., Ramkumar, N. and Anderson, K. V. (2019). p120-catenin regulates WNT signaling and EMT in the mouse embryo. Proc Natl Acad Sci U S A 116, 1687216881.

Holder, N. (1981). Regeneration and compensatory growth. Br Med Bull 37, 227-232.

Huangfu, D., Liu, A., Rakeman, A. S., Murcia, N. S., Niswander, L. and Anderson, K. V. (2003). Hedgehog signalling in the mouse requires intraflagellar transport proteins. Nature $\mathbf{4 2 6}$, 83-87.

Huelsken, J., Vogel, R., Brinkmann, V., Erdmann, B., Birchmeier, C. and Birchmeier, W. (2000). Requirement for beta-catenin in anterior-posterior axis formation in mice. J Cell Biol 148, 567-578.

Jain, D., Meydan, C., Lange, J., Claeys Bouuaert, C., Lailler, N., Mason, C. E., Anderson, K. V. and Keeney, S. (2017). rahu is a mutant allele of Dnmt3c, encoding a DNA methyltransferase homolog required for meiosis and transposon repression in the mouse male germline. PLoS Genet 13, e1006964.

Jain, R., Aggarwal, A. K. and Rechkoblit, O. (2018). Eukaryotic DNA polymerases. Curr Opin Struct Biol 53, 77-87.

Kinder, S. J., Tsang, T. E., Quinlan, G. A., Hadjantonakis, A. K., Nagy, A. and Tam, P. P. (1999). The orderly allocation of mesodermal cells to the extraembryonic structures and the anteroposterior axis during gastrulation of the mouse embryo. Development 126, 4691-4701.

615 Kojima, Y., Tam, O. H. and Tam, P. P. (2014). Timing of developmental events in the early mouse embryo. Semin Cell Dev Biol 34, 65-75. 
Kozar, K., Ciemerych, M. A., Rebel, V. I., Shigematsu, H., Zagozdzon, A., Sicinska, E., Geng, Y., Yu, Q., Bhattacharya, S., Bronson, R. T., et al. (2004). Mouse development and cell proliferation in the absence of D-cyclins. Cell 118, 477-491.

Kwon, G. S., Viotti, M. and Hadjantonakis, A. K. (2008). The endoderm of the mouse embryo arises by dynamic widespread intercalation of embryonic and extraembryonic lineages. Dev Cell 15, 509-520.

Lancey, C., Tehseen, M., Raducanu, V. S., Rashid, F., Merino, N., Ragan, T. J., Savva, C. G., Zaher, M. S., Shirbini, A., Blanco, F. J., et al. (2020). Structure of the processive human Pol delta holoenzyme. Nat Commun 11, 1109.

Lawson, K. A. (1999). Fate mapping the mouse embryo. Int J Dev Biol 43, 773-775.

Lawson, K. A., Meneses, J. J. and Pedersen, R. A. (1986). Cell fate and cell lineage in the endoderm of the presomite mouse embryo, studied with an intracellular tracer. Dev Biol 115, 325-339.

Lawson, K. A., Meneses, J. J. and Pedersen, R. A. (1991). Clonal analysis of epiblast fate during germ layer formation in the mouse embryo. Development 113, 891-911.

Lawson, K. A. and Pedersen, R. A. (1987). Cell fate, morphogenetic movement and population kinetics of embryonic endoderm at the time of germ layer formation in the mouse. Development 101, 627-652.

Lewis, N. E. and Rossant, J. (1982). Mechanism of size regulation in mouse embryo aggregates. J Embryol Exp Morphol 72, 169-181.

Lu, C. C., Brennan, J. and Robertson, E. J. (2001). From fertilization to gastrulation: axis formation in the mouse embryo. Curr Opin Genet Dev 11, 384-392.

Martinez-Barbera, J. P., Rodriguez, T. A. and Beddington, R. S. (2000). The homeobox gene Hesx 1 is required in the anterior neural ectoderm for normal forebrain formation. Dev Biol 223, 422-430.

Migeotte, I., Grego-Bessa, J. and Anderson, K. V. (2011). Rac1 mediates morphogenetic responses to intercellular signals in the gastrulating mouse embryo. Development 138 , 3011-3020.

Mishina, Y., Suzuki, A., Ueno, N. and Behringer, R. R. (1995). Bmpr encodes a type I bone morphogenetic protein receptor that is essential for gastrulation during mouse embryogenesis. Genes Dev 9, 3027-3037.

Murphy, M., Stinnakre, M. G., Senamaud-Beaufort, C., Winston, N. J., Sweeney, C., Kubelka, M., Carrington, M., Brechot, C. and Sobczak-Thepot, J. (1997). Delayed early 
embryonic lethality following disruption of the murine cyclin A2 gene. Nat Genet 15, 8386.

Power, M. A. and Tam, P. P. (1993). Onset of gastrulation, morphogenesis and somitogenesis in mouse embryos displaying compensatory growth. Anat Embryol (Berl) 187, 493-504.

Rivera-Perez, J. A., Mager, J. and Magnuson, T. (2003). Dynamic morphogenetic events characterize the mouse visceral endoderm. Dev Biol 261, 470-487.

Rodriguez, T. A., Casey, E. S., Harland, R. M., Smith, J. C. and Beddington, R. S. (2001). Distinct enhancer elements control Hex expression during gastrulation and early organogenesis. Dev Biol 234, 304-316.

Rossant, J. and Tam, P. P. (2009). Blastocyst lineage formation, early embryonic asymmetries and axis patterning in the mouse. Development 136, 701-713.

Sasaki, H., Yanagi, K., Ugi, S., Kobayashi, K., Ohkubo, K., Tajiri, Y., Maegawa, H., Kashiwagi, A. and Kaname, T. (2018). Definitive diagnosis of mandibular hypoplasia, deafness, progeroid features and lipodystrophy (MDPL) syndrome caused by a recurrent de novo mutation in the POLD1 gene. Endocr J 65, 227-238.

Shimono, A. and Behringer, R. R. (2003). Angiomotin regulates visceral endoderm movements during mouse embryogenesis. Curr Biol 13, 613-617.

Skarnes, W. C., Rosen, B., West, A. P., Koutsourakis, M., Bushell, W., lyer, V., Mujica, A. O., Thomas, M., Harrow, J., Cox, T., et al. (2011). A conditional knockout resource for the genome-wide study of mouse gene function. Nature 474, 337-342.

Snow, M. H. and Tam, P. P. (1979). Is compensatory growth a complicating factor in mouse teratology? Nature 279, 555-557.

Srinivas, S., Rodriguez, T., Clements, M., Smith, J. C. and Beddington, R. S. (2004). Active cell migration drives the unilateral movements of the anterior visceral endoderm. Development 131, 1157-1164.

Stanger, B. Z., Tanaka, A. J. and Melton, D. A. (2007). Organ size is limited by the number of embryonic progenitor cells in the pancreas but not the liver. Nature 445, 886-891.

Summerbell, D. (1981). Evidence for regulation of growth, size and pattern in the developing chick limb bud. J Embryol Exp Morphol 65 Suppl, 129-150.

Tam, P. P., Khoo, P. L., Lewis, S. L., Bildsoe, H., Wong, N., Tsang, T. E., Gad, J. M. and Robb, L. (2007). Sequential allocation and global pattern of movement of the definitive endoderm in the mouse embryo during gastrulation. Development 134, 251-260.

Uchimura, A., Hidaka, Y., Hirabayashi, T., Hirabayashi, M. and Yagi, T. (2009). DNA polymerase delta is required for early mammalian embryogenesis. PLoS One 4, e4184. 
Venkatesan, R. N., Treuting, P. M., Fuller, E. D., Goldsby, R. E., Norwood, T. H., Gooley, T.

A., Ladiges, W. C., Preston, B. D. and Loeb, L. A. (2007). Mutation at the polymerase active site of mouse DNA polymerase delta increases genomic instability and accelerates tumorigenesis. Mol Cell Biol 27, 7669-7682.

Vincent, S. D., Dunn, N. R., Hayashi, S., Norris, D. P. and Robertson, E. J. (2003). Cell fate decisions within the mouse organizer are governed by graded Nodal signals. Genes Dev 17, 1646-1662.

Weedon, M. N., Ellard, S., Prindle, M. J., Caswell, R., Lango Allen, H., Oram, R., Godbole, K., Yajnik, C. S., Sbraccia, P., Novelli, G., et al. (2013). An in-frame deletion at the polymerase active site of POLD1 causes a multisystem disorder with lipodystrophy. Nat Genet 45, 947-950.

695 Winnier, G., Blessing, M., Labosky, P. A. and Hogan, B. L. (1995). Bone morphogenetic protein-4 is required for mesoderm formation and patterning in the mouse. Genes Dev 9 , 2105-2116.

Zhou, X. and Anderson, K. V. (2010). Development of head organizer of the mouse embryo depends on a high level of mitochondrial metabolism. Dev Biol 344, 185-195. 


\section{Figure Legends}

Figure 1. Characterization of tiny siren (tyrn), a mutant recovered from an ENU Screen. (A) Top: Wildtype and tym embryos recovered at E8.5. Embryos were aligned as they were in decidua. Mutant embryos showed a shifted body A-P axis, with a small, unstructured head located at the distal tip (arrowhead) and the tail at the posterior-proximal side (arrow). Scale Bar $=500 \mu \mathrm{m}$. Bottom: A cartoon that shows the orientation of wildtype and mutant embryos inside decidua. (B) Foxg1 labels forebrain and (C) Otx2 labels midbrain and forebrain in wildtype embryos. Mutant embryos expressed both Foxg1 (B) and Otx2 (C) in head. (D)T (Brachyury) marks the primitive streak and notochord in E8.5 wild types and tyrn. (E) Both wild types and tyrn expressed Foxa2 in the floor plate, posterior notochord, notochordal plate and gut endoderm. $\mathrm{n}=3$ embryos per genotype. Scale Bar $=200 \mu \mathrm{m}$.

Figure 2. Identification of the Pold1 missense mutation in tyrn. (A) Schematic diagrams of the murine Pold1 locus in genome (upper panel) and of the Pold1 domain structure (lower panel). The G2815T nucleotide change (red arrow) was located in exon 23. The corresponding D939Y amino acid substitution was located in the DNA polymerase domain close to the Cterminal domain (CTD). (B) Multiple alignment of orthologous Pold1 amino acid sequences around D939Y among different eukaryotic organisms. The mutated aspartate residue was highly conserved. (C) Pold1 expression level in wildtype (left lane) and tyrn (right lane) embryos at E8.5 shown by western blot. (D) Complementation test crossing strategy (upper panel). Wildtype and tyrn/tm $1 \mathrm{~b}$ embryos acquired at E7.5 from the complementation test (lower panel). tyrn/tm1b embryos did not undergo gastrulation and were not able to survive past E7.5. (E) Crossing strategy to harvest homozygous mutants from heterozygous mice carrying tyrn allele (upper panel). E7.5 wildtype and mutant embryos (lower panel). $n=3$ per genotype. Scale Bar $725=100 \mu \mathrm{m}$.

Figure 3. The effects of the D941Y mutation in Po/3 on DNA synthesis. (A) Workflow of the in vitro primer extension assay. (B) Denatured protein gel showing the overexpressed yeast Pol $\delta^{\mathrm{WT}}$ and Pol $\delta^{\mathrm{D} 941 \mathrm{Y}}$. The Pol3 ${ }^{\mathrm{WT}}$ and Pol3 ${ }^{\mathrm{D} 941 \mathrm{Y}}$ corresponds to mouse Pold $1^{\mathrm{WT}}$ and Pold1 ${ }^{\mathrm{D} 939 \mathrm{Y}}$, respectively. The Pol31 and Pol32 are the associated subunits in Pol $\delta$ corresponding to Pold 2 and Pold3 in mouse. (C) In vitro primer extension assay showing reduction of DNA synthesis efficiency of Pol3 ${ }^{\mathrm{D} 941 Y}$, the yeast orthologue which harbors the corresponding D939Y mutation identified in mouse. (D) Nonlinear-fitted curves of total amount of new synthesized DNAs at different time points. The mean is represented by black squares (Pol $\delta^{\mathrm{WT}}$ ) and grey squares (Pol 
$735 \delta^{\text {D941Y }}$ ). Error bars represent s.e.m.. For each time point, $n=3$ per genotype. (E) Nonlinear-fitted curves of the percentage of full-length circular DNAs among total products. The mean is represented by black squares (Pol $\delta^{\mathrm{WT}}$ ) and grey squares (Pol $\left.\delta^{\mathrm{D} 941 \mathrm{Y}}\right)$. Error bars represent s.e.m.. For each time point, $n=3$ per genotype. Multiple Student's $t$ test, two-tailed, $p<0.05$.

Figure 4. Analysis of DNA synthesis and cell proliferation in tyrn mutants. (A) EdU incorporation levels in wildtype and mutant embryos at E6.0, E6.5 and E7.0. $n=3$ embryos per genotype. Scale Bar = $100 \mu \mathrm{m}$. (B). Quantification of EdU signal intensity showing by box whisker plot. EdU signals of the embryos at the same stage was normalized to the maximum intensity in that stage. Total embryos quantified per genotype: $n=3$. Unpaired Student's $t$ test, two tailed, $p<0.0001$. Within box plot, the median is represented by the horizontal dividing line and the top and bottom of the box represents the seventy-fifth and twenty-fifth percentile, with the whiskers indicating the maximum and minimum points. (C) Curves of total cell number of wildtype and tyrn embryo at E6.0, E6.5 and E7.0 stages. n=3 embryos per genotype per stage. Unpaired Student's $t$ test, two-tailed, $p<0.001$. Within the curve, the mean is represented by the black squares (wildtype) and grey squares (tyrn). the error bars represent s.d.. (D) Immunofluorescence staining of Cleaved Caspase-3 in wildtype and mutant embryos at E7.5. $\mathrm{n}$ $=3$ per genotype. Scale Bar $=50 \mu \mathrm{m}$.

Figure 5. Position of Anterior Visceral Endoderm in tyrn mutants. Cerl (A) and Dkk1 (B) were expressed in anterior visceral endoderm (AVE) in both wildtype and tyrn embryos at E6.5. (C) Hhex-GFP was expressed in AVE in both wildtype and tyrn embryos at E6.5. $n=3$ embryos per genotype. Scale Bar $=50 \mu \mathrm{m}$.

\section{Figure 6. Characterization of primitive streak extension in tyrn mutants at E7.5. Co-} staining of Sox2 with Foxa2 (A) or with $T(B)$. Co-staining of with $T(C)$ or Foxa2 (D). The anterior marker Sox2 was strongly expressed at the distal tip in mutants. Sox2 was also expressed in the ectoderm of chorion in both wildtype and mutant embryos. A short primitive streak was formed. AME and ADE cells emerged from the midpoint of posterior side. Sox2 labels the extraembryonic ectoderm of the chorion. (E) in situ hybridization staining of Otx2 showed that the Otx2 expression was restricted distally in tyrn rather than anteriorly seen in wild types. (F) Foxa2 labels axial mesoderm and definitive endoderm cells. (G) Paraxial mesoderm marker Tbx6 expression was restricted to the posterior, consistent with the T expression pattern. $(\mathrm{H})$ Twist was first expressed in extraembryonic mesoderm and then strongly expressed 
in allantois. It was also expressed in embryonic mesoderm which leaves primitive streak. (I)

Tbx4 expression in allantois was strong in wild types but not seen mutant embryos at E7.5. $\mathrm{n}=$ 3 embryos per genotype. Scale Bar $=100 \mu \mathrm{m}$.

\section{Figure 7. Developmental progression of wildtype and tyrn embryos between E6.5 and}

\section{E8.5. At E6.5, wildtype and tyrn embryos are morphologically indistinguishable. AVE (red) is}

properly localized at the normal anterior region in both wildtype and mutant embryos.

Gastrulation is initiated and primitive streak is formed (yellow). At E7.0. primitive streak elongation (yellow) in tyrn shows slight delay compared to wildtype embryos. Embryo size also starts to show difference although still not visually obvious. At E7.5, the primitive streak extension in tyrn was delayed. The AME and ADE emerged from the midpoint of the posterior side and did not migrate across the bottom point of the embryos (magenta). The distal tip in mutants becomes the anterior signal center and embryos consider the distal tip as the place for head induction and morphogenesis. At E8.5, the wildtype embryos are properly developed along normal A-P axis, whereas the mutant embryos exhibit a small, siren-like morphology with head pointing to the bottom of the embryo. The asterisk indicates the reduction of extraembryonic mesoderm cells in the allantois.

\section{Supplementary Figure 1. Summary of Pold1 mutant mice and crossing strategy of} generating Pold1 null allele. (A) Schematic domain structure in mouse Pold1. The red bars represent mutations generated in previous genetic studies documented in the literature. The yellow bar denotes the missense mutation identified from ENU mutagenesis screen in this study. (B) Structure of the Pold1 tm1a (gene trap) allele and the tm1b (null) allele generated after CAG-Cre-mediated recombination. Exons are represented in grey vertical blocks.

\section{Supplementary Figure 2. Cryo-EM structure of human POLD1 associated with DNA} duplex and PCNA. (A) Structure of the POLD1-DNA-PCNA complex colored by the domain and sequence of the DNA. (B) Zoom-in image showing the POLD1 thumb domain (red). The mutated Asp 941(D941) corresponsive to mouse Asp 939 (D939) residue was highlighted in lemon. (C) Zoom-in image showing the POLD1 thumb domain. The mutated Asp 941(D941) residue was shown as lemon stick. The protein structure information was extracted from protein data bank (PDB). Structure ID: 6TNY. PDB DOI: 10.2210/pdb6TNY/pdb. The original protein structure was adapted using PyMOL to deliver information relevant to this study. 
Supplementary Figure 3. Cell apoptosis in E6.5 embryos. Whole mount staining of E6.5 wildtype and tyrn embryos. Cleaved Caspase-3 (Red) stained for apoptotic cells. DAPI (blue) stained nucleus of all cells. $n=3$ per genotype. Scale Bar $=100 \mu \mathrm{m}$. 

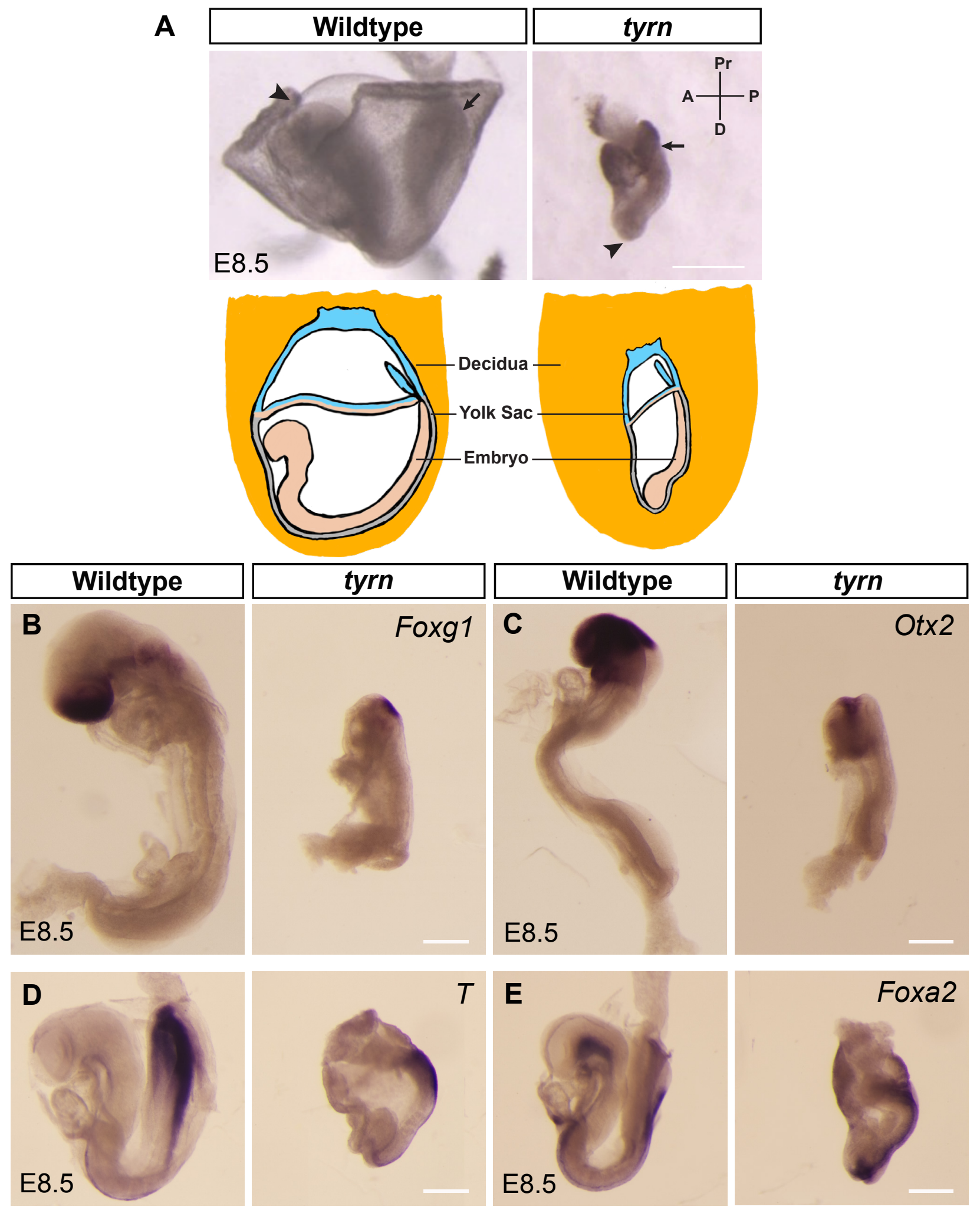
A

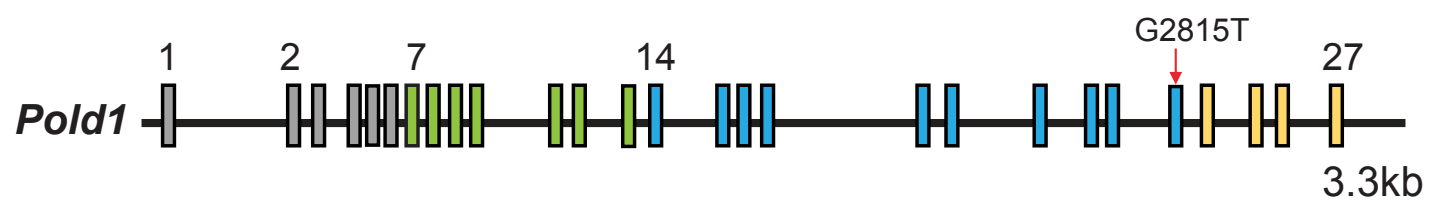

Pold1

$$
\text { exo }
$$

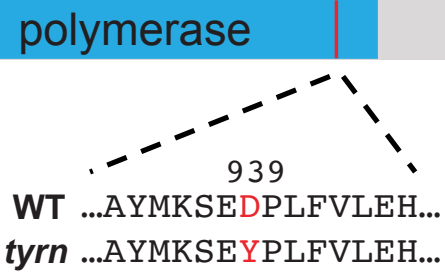

CTD

1105aa

B

H. sapiens

933 VAAYMKSEDPLFVLEHSLP 951

931 VAAYMKSEDPLFVLEHSLP 949

M. musculus

D. melanogaster 919 TPAYQKAEDPLYVLENSVP 938

C. elegans

903 VPAYERAEDPTFVLQNNIP 921

S. cerevisiae

\section{DKLYNRAEDPLFVLENNIQ 951}

C

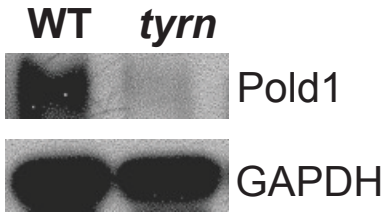

D

Complementation Test

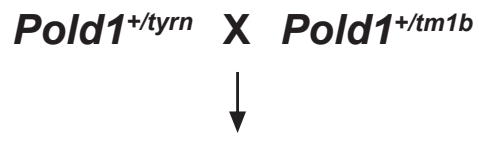

Pold1 ${ }^{+/+}$Pold1+/tyrn Pold1 ${ }^{+/ t m 1 b}$ Pold1tyrn/tm1b Wildtype

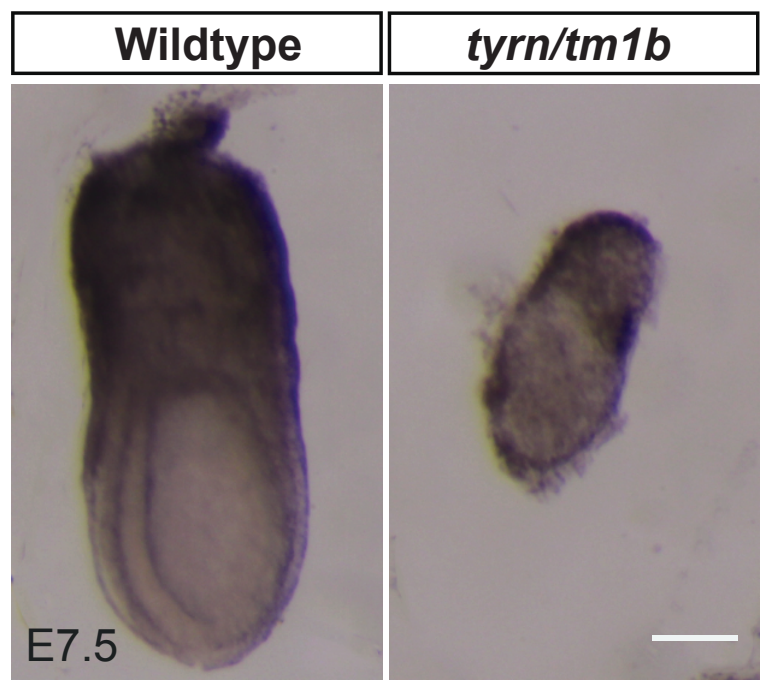

E ENU Crossing Pold1 $1^{+/ t y r n} \times$ Pold1+/tyrn $\downarrow$

Pold1 $^{+/+}$Pold1+/tyrn Pold1 ${ }^{+/ t y r n}$ Pold1tyrn/tyrn Wildtype

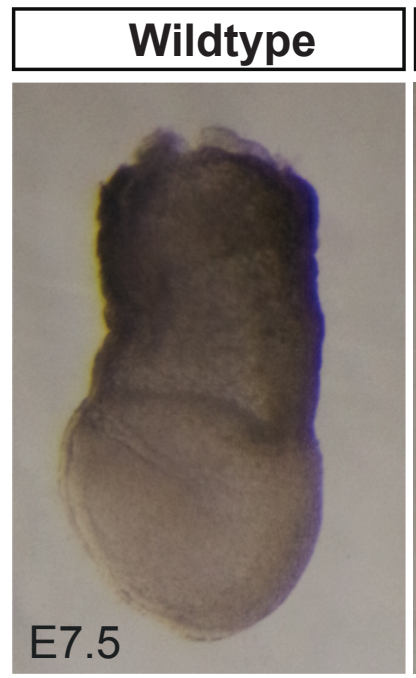

tyrn/tyrn 
A

Primer Extension Assay

Primed SsM13,ATP, RFC, PCNA, RPA, Pol $\delta$ dCTP,dGTP, $\alpha^{32} P-d A T P$

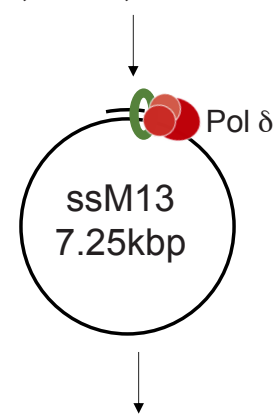

dTTP

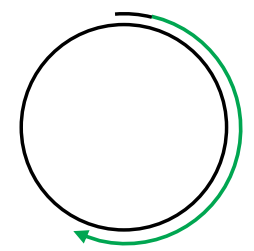

B

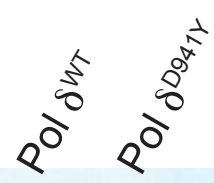

D

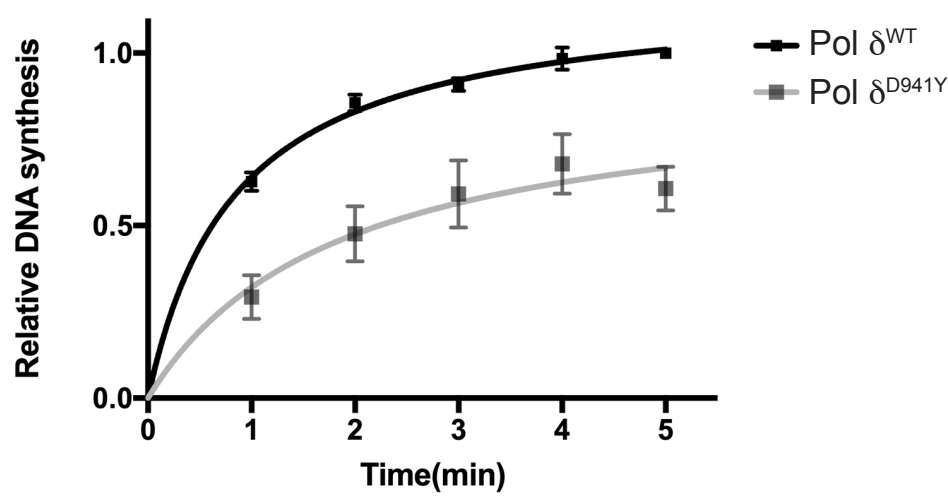

C

$$
\text { Pol3 }{ }^{\mathrm{D} 941 \mathrm{Y}} \text { Pol3WT }
$$

Time (min) 122345512345

$\underline{k b p}$

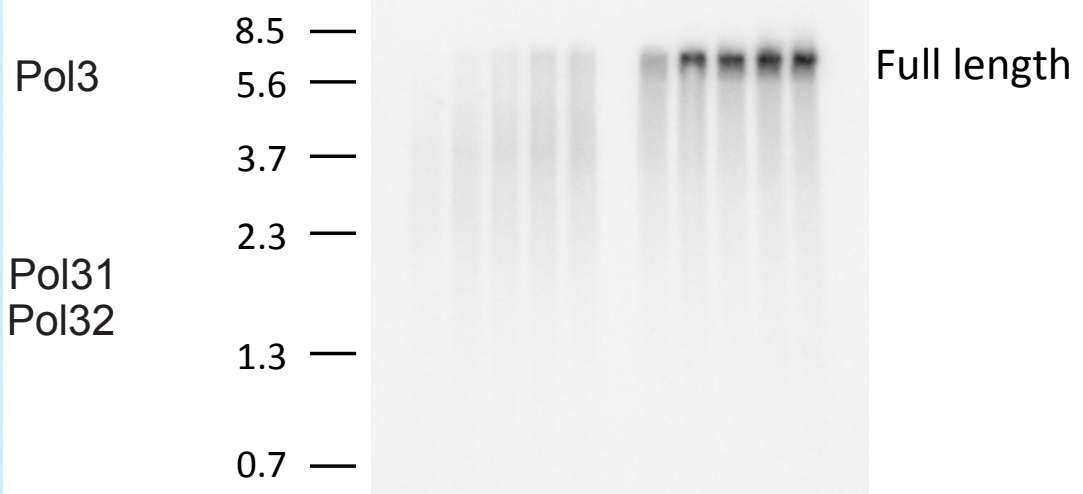

E

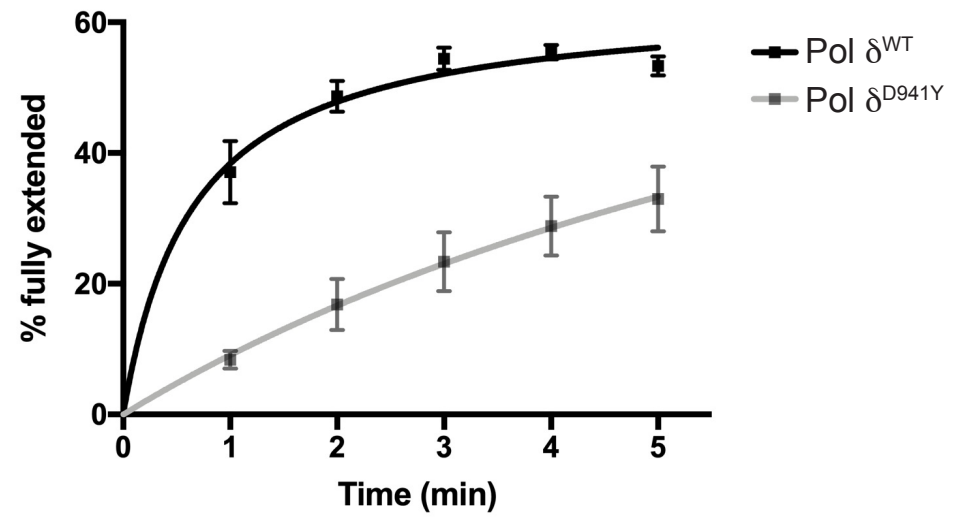


A
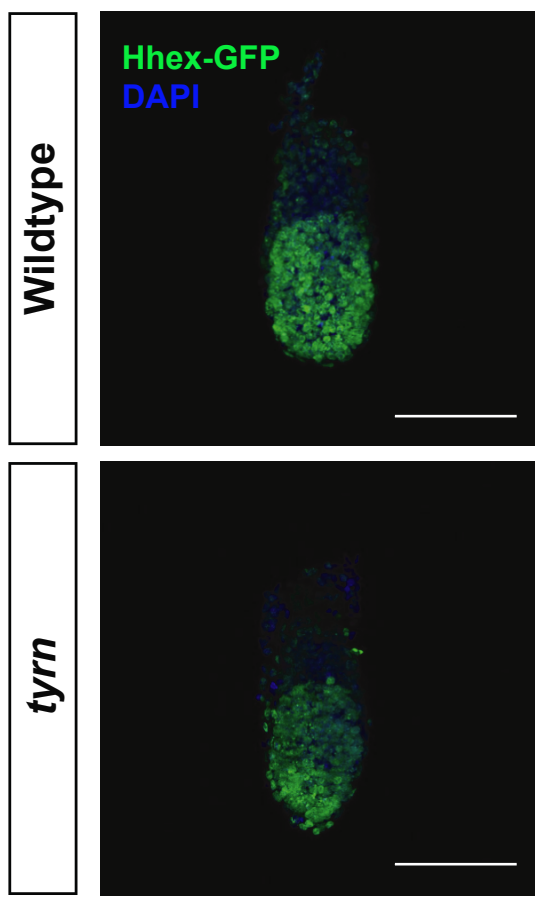

B

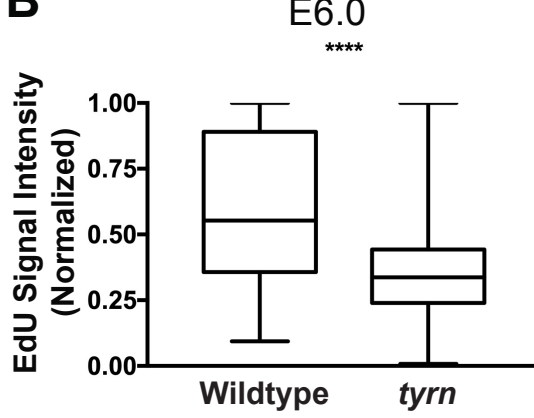

C

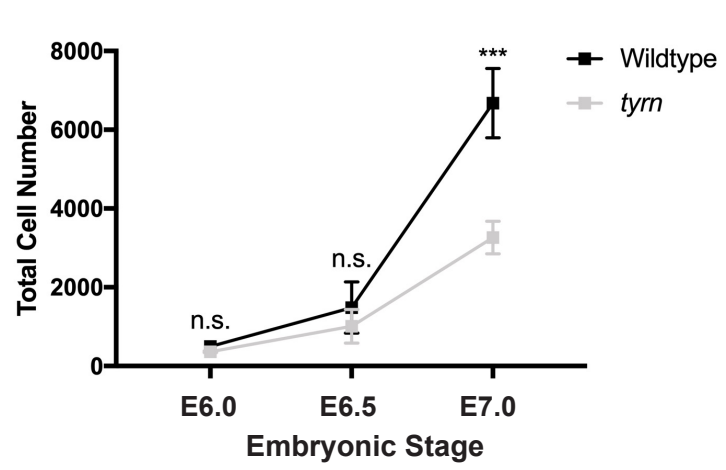

E6.5

E7.0
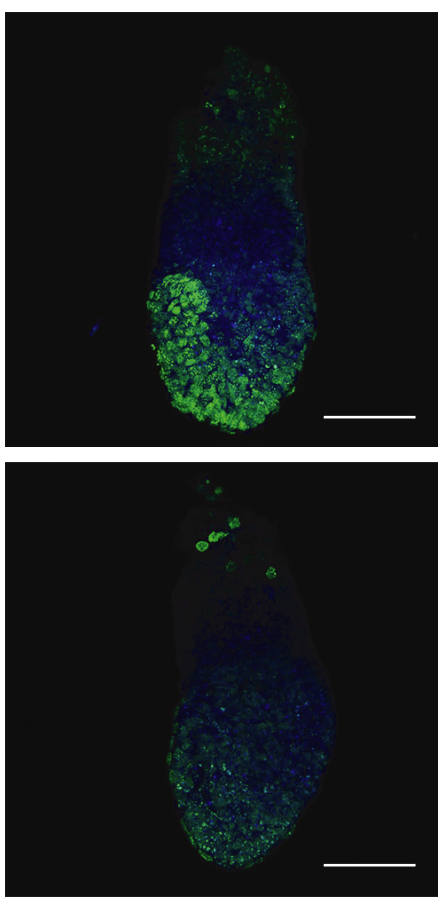

E6.5

****
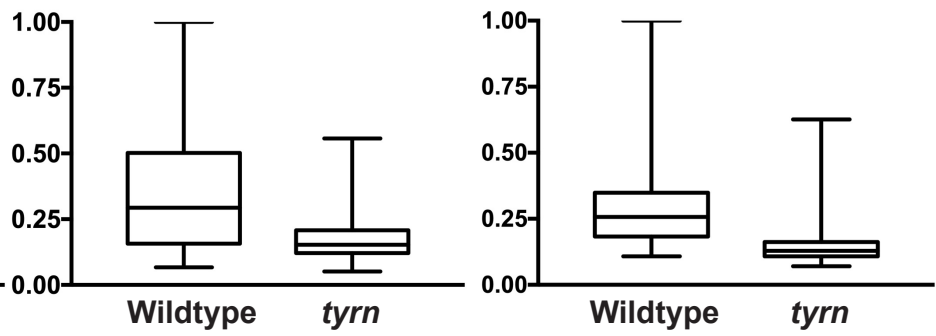

D

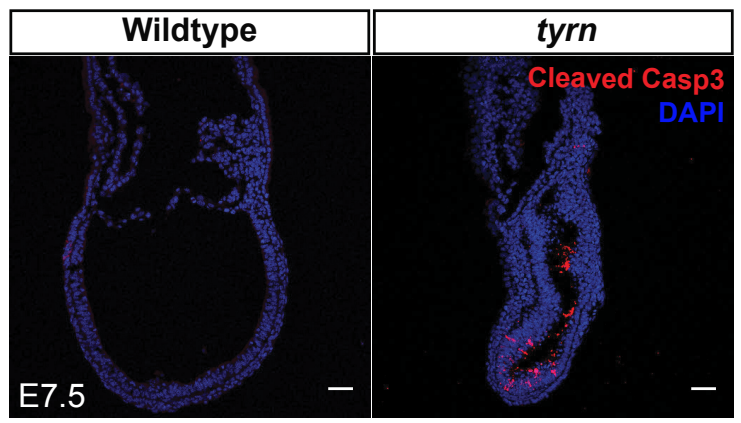




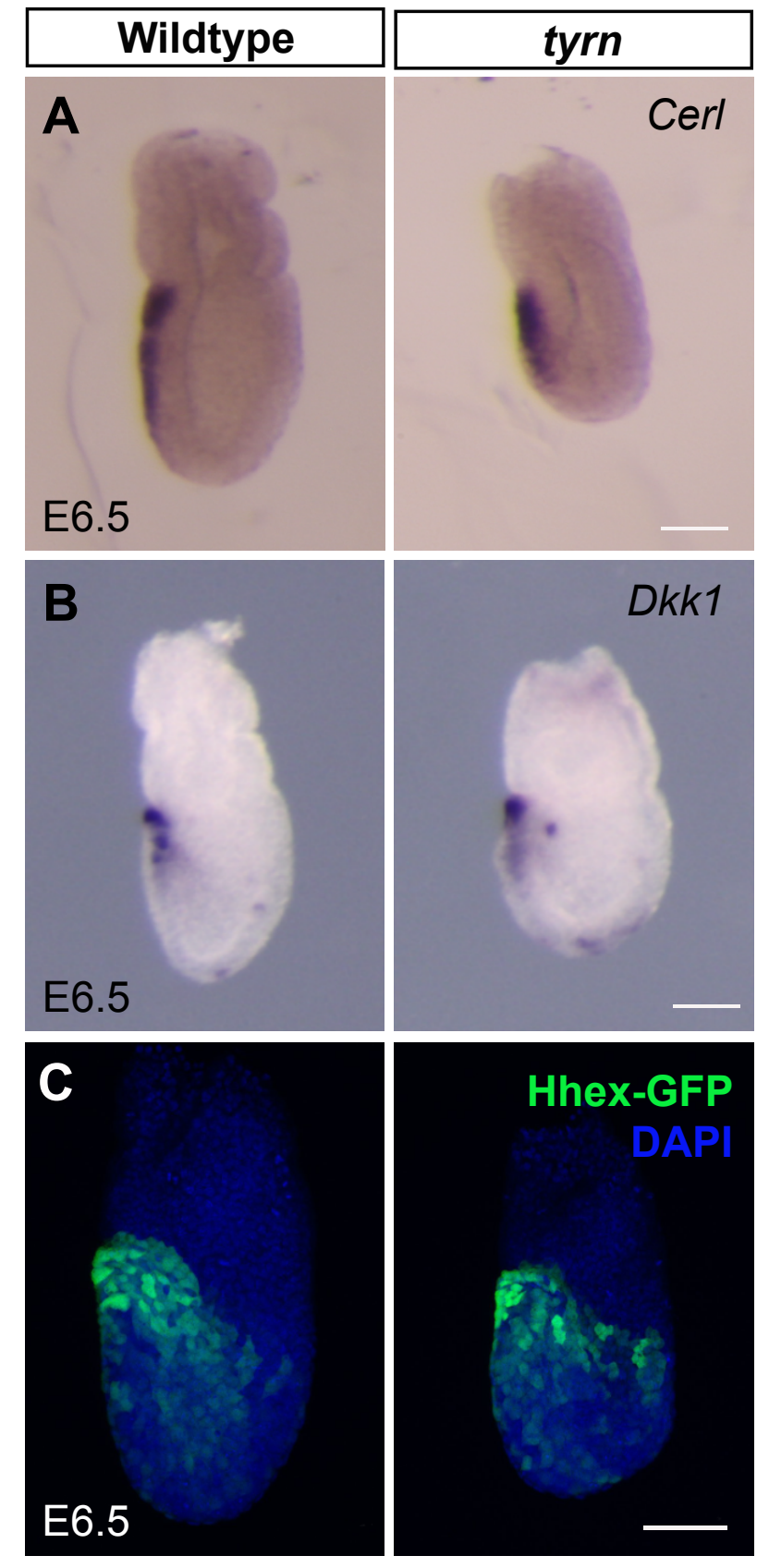




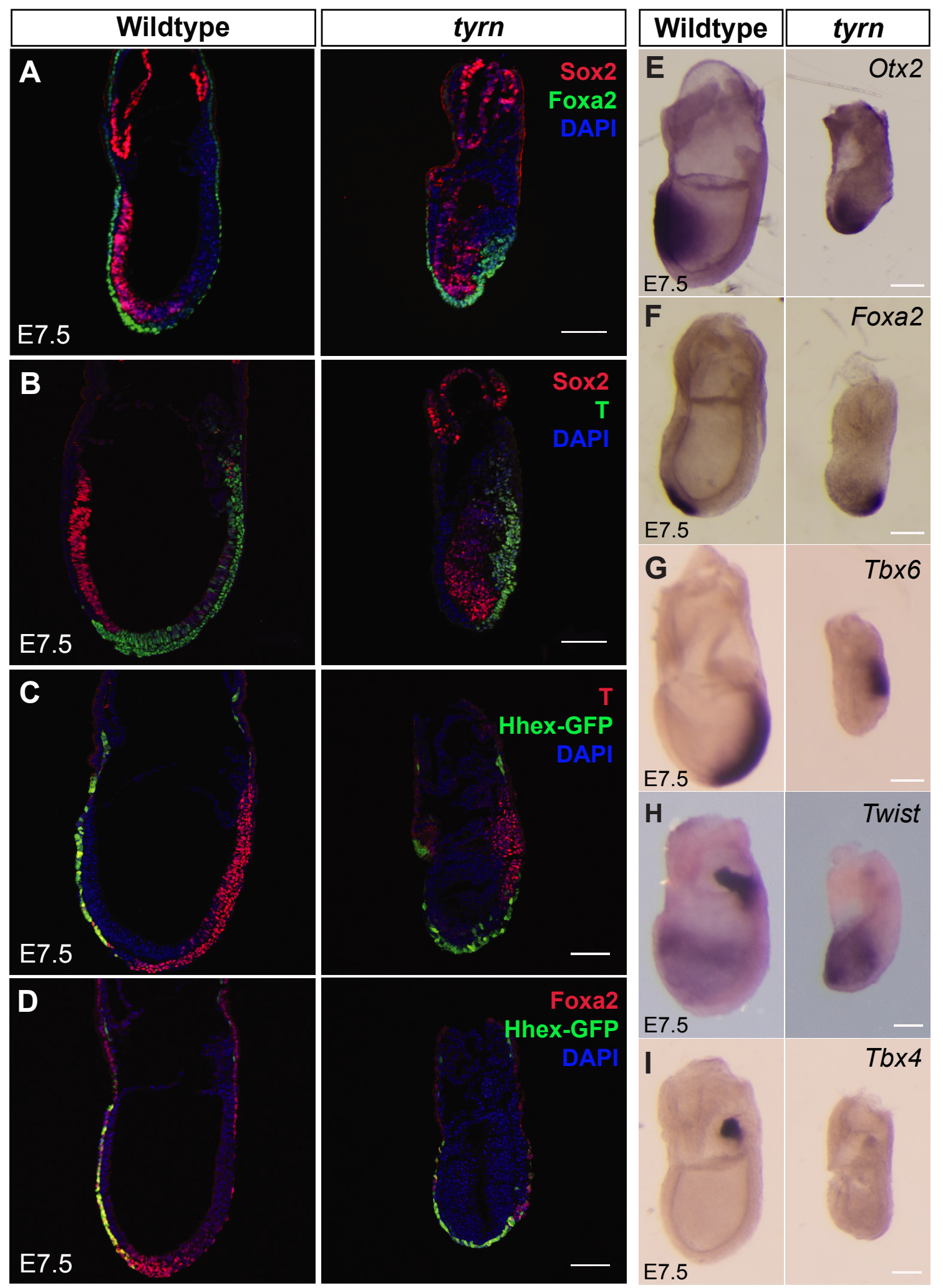




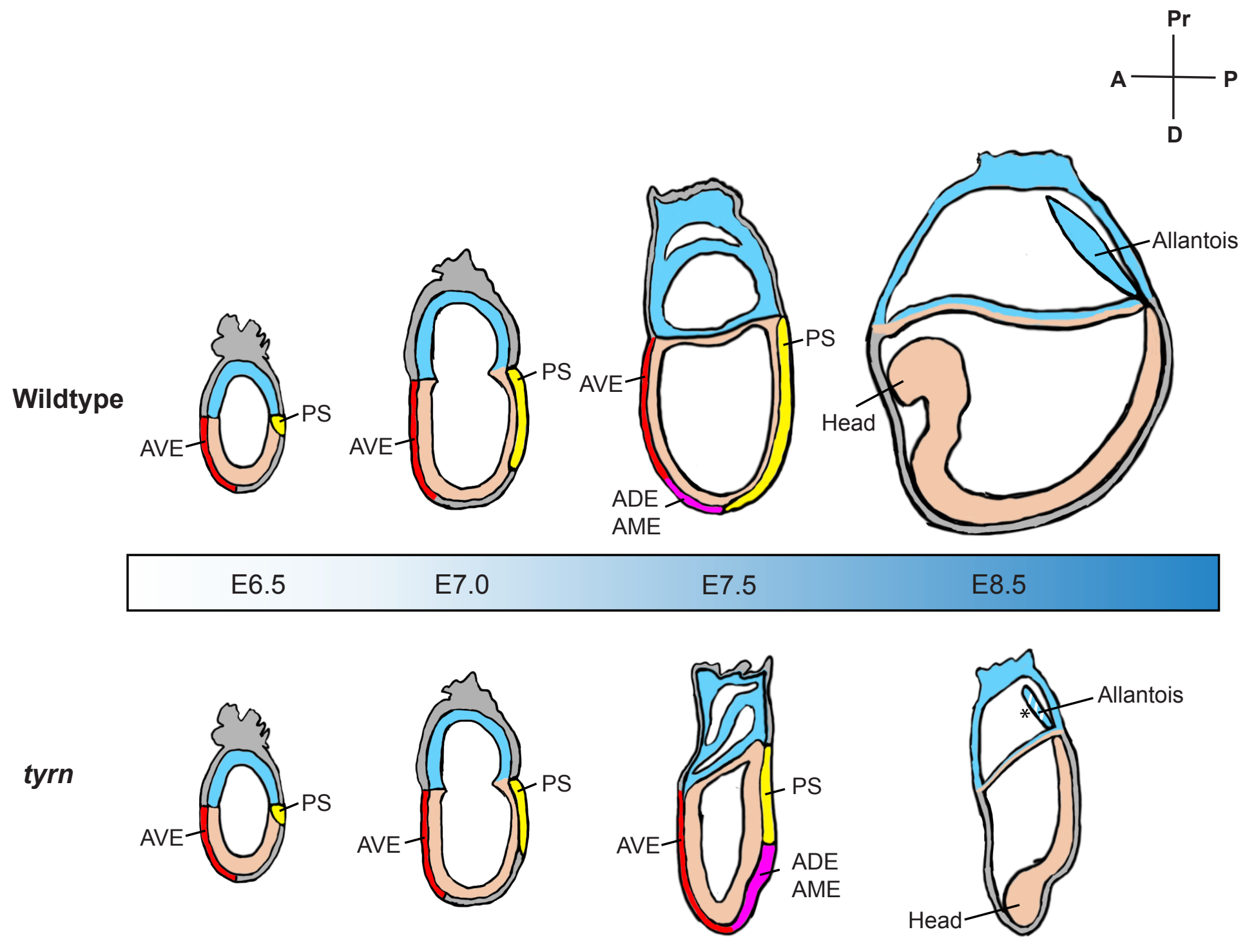

